\title{
Autophagy induction by low-dose cisplatin: The role of p53 in autophagy
}

\author{
KYUNG-HWA CHO ${ }^{1}$, JI-HYE PARK ${ }^{1}$, KANG-BEOM KWON $^{2}$, YOUNG-RAE LEE ${ }^{2}$, HONG-SEOB SO ${ }^{2}$, \\ KANG-KYOO LEE ${ }^{3}$, SAM-YOUN LEE ${ }^{4}$, SUN-ROCK MOON ${ }^{3}$ and SEI-HOON YANG ${ }^{1}$ \\ ${ }^{1}$ Department of Internal Medicine; ${ }^{2}$ Center for Metabolic Function Regulation; Departments of ${ }^{3}$ Radiology and \\ ${ }^{4}$ Thoracic Surgery, Wonkwang University School of Medicine, Iksan, Jeonbuk, Republic of Korea
}

Received August 5, 2013; Accepted September 20, 2013

DOI: $10.3892 /$ or.2013.2809

\begin{abstract}
The majority of chemotherapy treatments for lung cancer use cisplatin; however, its use is limited as it has several side-effects. Autophagy (or type II cell death) is an important mechanism by which programmed cell death occurs. The purpose of this study was to determine whether low-dose cisplatin treatment induces autophagy in lung cancer cells. We also examined whether autophagy inhibition results in p53-mediated apoptosis. NCI-H460 (wild-type p53) and NCI-H1299 (null-type p53) cells were treated with 5 or $20 \mu \mathrm{M}$ cisplatin for 12, 24 or $48 \mathrm{~h}$. An MTT assay was performed to measure the cell viability following cisplatin treatment. To detect cisplatin-induced autophagy, cell morphology (autophagic vacuole) and LC3 localization were examined. The outcome of autophagy inhibition was determined using 3-methyladenine (3-MA) to detect Annexin V (+), propidium iodide (PI) (-) and acridine orange (+) cells by FACS analysis. To determine whether cisplatin induced autophagy, we examined the role of p53 as a cell survival regulator in autophagy. Low-doses of cisplatin $(5 \mu \mathrm{M})$ induced cell death and this was augmented by 3 -MA in both cell lines. Autophagic vacuoles and cytoplasmic LC3 formation was more evident in H460 cells than in H1299 cells. The induction of autophagy by low-dose cisplatin was increased by 2 -fold in H460 compared to $\mathrm{H} 1299$ cells. However, the tests for apoptosis showed no difference between the 2 cell lines. Following 3-MA pretreatment, cisplatin-induced autophagy was found to be markedly reduced (a 3-fold reduction) in wild-type p53 compared to nulltype 553 cells. However, cisplatin-induced apoptosis increased in wild-type p53 compared to null-type p53 cells. Autophagy induction and apoptotic shift after autophagy inhibition may be mediated by p53 activation in lung cancer cells treated with low-dose cisplatin.
\end{abstract}

Correspondence to: Professor Sei-Hoon Yang, Department of Internal Medicine, Wonkwang University School of Medicine, 344-2 Shinyong-dong, Iksan, Jeonbuk 570-711, Republic of Korea E-mail: yshpul@wku.ac.kr

Key words: autophagy, apoptosis, p53, low-dose cisplatin

\section{Introduction}

Lung cancer treatment continues to present difficulties and survival rates remain low, despite research efforts. Chemotherapy is the primary form of treatment for lung cancer; however, its response rate is low, and complete recovery is rare $(1,2)$. Thus, the development of a more efficacious therapy for lung cancer is urgently required. Autophagy is a type of programmed cell death that occurs in both physiological or pathophysiological environments $(3,4)$. Autophagy is the mechanism by which protein conversion and the removal of aging or damaged organelles occurs; this helps maintain cellular homeostasis $(5,6)$. Autophagy is also known to be involved in cell survival. However, excessive autophagy or improper activation can lead to apoptosis. Autophagy is believed to play an important role in the incidence of tumors; it is also considered to have a role in primary tumorigenesis. Additionally, it is known to maintain cell survival in tumors via survival pathways during periods of stress, such as during tumor progression or chemotherapy (7-9). The protein p53 is an 'intracellular gatekeeper' that protects the cell from various stress signals, and p53 is a mutant gene in most cancer cells that is activated by DNA damage, cancer gene activation, hypoxia and other stresses $(10,11)$. It is involved in cell cycle inhibition, apoptosis, aging, metabolism, differentiation, inhibition of blood vessel formation, and autophagy regulation (12-14). Cisplatin is the most widely used chemotherapy drug for lung cancer and has powerful anticancer effects. However, it has many side-effects. Therefore, in an attempt to reduce these side-effects, we confirmed the anticancer effect of low-dose cisplatin, and examined the effects of these low-doses on autophagy and apoptosis. To ascertain whether p53 influences autophagy and apoptosis, we compared the role of p53 in lung cancer cell lines of wild-type p53 and null-type p53.

\section{Materials and methods}

Cell lines. The wild-type p53 NCI-H460 and null-type p53 NCI-H1299 lung cancer cell lines were purchased from the American Type Culture Collection (ATCC, Manassas, VA, USA).

Reagents. RPMI-1640, antibiotics, trypsin and fetal bovine serum (FBS) were obtained from Gibco-BRL (Grand Island, 
NY, USA). 24- and 48-well plates, along with 6 and 10-cm diameter dishes, were purchased from Nunc (Thermo Fisher Scientific, Roskilde, Denmark). Cisplatin $(0.5 \mathrm{mg} / \mathrm{ml})$ was obtained from Ildong Pharmaceutical Co., Ltd. (Seoul, Korea). Propidium iodide (PI), 3-methyladenine (3-MA), 3-(4,5-dimethylthiazol-2-yl)-2,5-diphenyltetrazolium bromide (MTT), and acridine orange (AO) were purchased from Assay Designs (Ann Arbor, MI, USA). Glyceraldehyde-3phosphate dehydrogenase (GAPDH) antibody and p53 were obtained from Santa Cruz Biotechnology, Inc. (Santa Cruz, CA, USA). Secondary antibody was obtained from Amersham (Buckinghamshire, UK). Polyvinylidene fluoride (PVDF) membrane, and enhanced chemiluminescent (ECL) kit were purchased from Millipore Co. (Billerica, MA, USA).

Cell culture. NCI-H460 and NCI-H1299 cells were cultured with RPMI medium supplemented with $10 \%$ FBS, $1 \%$ antibiotics, and 4-(2-hydroxyethyl)-1-piperazineethanesulfonic acid (HEPES). Cells were incubated in $5 \% \mathrm{CO}_{2}$ at $37^{\circ} \mathrm{C}$. The medium was replaced every $24 \mathrm{~h}$. Experiments were conducted with cells in the log phase. The viability of the cultured cells was measured after $24 \mathrm{~h}$ of treatment with cisplatin at $5 \mu \mathrm{M}$ (LD20) and $20 \mu \mathrm{M}$ (LD50).

Cell viability. Cell viability was determined with MTT assays. Cells were seeded in 24-well plates at a density of $1 \times 10^{4}$ cells and incubated for $24 \mathrm{~h}$. After treatment with cisplatin $(5 \mu \mathrm{M})$ in the presence or absence of 3-MA $(10 \mathrm{mM})$, MTT $(5 \mathrm{mg} / \mathrm{ml})$ was added to each well and incubated in $5 \% \mathrm{CO}_{2}$ at $37^{\circ} \mathrm{C}$ for 4 h. Crystals were dissolved in $200 \mu 1$ dimethyl sulfoxide (DMSO). The absorbance of the solution was measured spectrophotometrically at $570 \mathrm{~nm}$ with a microplate ELISA reader (Thermo Scientific). The absorbance of formazan formed in control cells was considered as showing $100 \%$ cell viability, and the positively stained cells with MTT were expressed as the percentage of control cells.

Morphological observations. To observe the cell morphology after cisplatin treatment, H460 and H1299 cells were cultured in a 24 -well plate. They were then treated with 5 and $20 \mu \mathrm{M}$ cisplatin for 24 and $48 \mathrm{~h}$. After the treatment, the medium was removed and washed twice with phosphate buffered saline (PBS) (pH 7.4). It was then treated with $300 \mu$ l crystal violet solution $(0.05 \%$ crystal violet, $3.7 \%$ paraformaldehyde) for $5 \mathrm{~min}$ at room temperature and then washed twice with PBS. The nucleus and the cytoplasm were then observed under a microscope (Olympus, Japan).

Assay for autophagy detection. Autophagy was detected by measuring the expression levels of LC3-II protein and by using AO stain to detect acidic vesicular organelles (AVOs) within the cytoplasm. After adding AO $(1 \mu \mathrm{g} / \mathrm{ml})$, the cells were incubated in $5 \% \mathrm{CO}_{2}$ at $37^{\circ} \mathrm{C}$ for $15 \mathrm{~min}$. Next, the cells were washed with PBS. The number of AO-stained cells was observed under a microscope (Olympus, Japan).

LC3 protein was detected by western blot analysis. The cells were treated with cisplatin $(5 \mu \mathrm{M})$ in the presence or absence of 3-MA (10 mM) for $24 \mathrm{~h}$. After treatment, each cell was harvested and washed twice with ice-cold PBS and lysed in lysis buffers (50 mM HEPES, pH 7.4, $150 \mathrm{mM} \mathrm{NaCl}, 1 \%$ deoxycholate, $1 \mathrm{mM}$ EDTA, $1 \mathrm{mM}$ PMSF, $1 \mu \mathrm{g} / \mathrm{ml}$ aprotinin). After incubation for $1 \mathrm{~h}$ on ice, the cells were centrifuged at $10,000 \mathrm{rpm}$ for $30 \mathrm{~min}$ at $4^{\circ} \mathrm{C}$, and the supernatants were collected. The protein concentration was determined using the Bradford method. For western blot analysis, equal amounts of total protein were loaded onto 10 or $15 \%$ sodium dodecyl sulfate-polyacrylamide gel electrophoresis (SDS-PAGE) and then transferred onto a PVDF membrane. The PVDF membrane was blocked with 5\% skimmed milk in PBS for 20-90 min. After washing in PBS, immunoblots were analyzed using specific primary antibodies. The membrane was then washed with PBS and treated with secondary antibodies for $1 \mathrm{~h}$. Proteins were then visualized using an ECL kit.

Quantitative analysis of apoptosis and autophagy. To quantitatively analyze apoptosis and autophagy, the cells were plated in 6-well plates and incubated for $24 \mathrm{~h}$. They were then treated with cisplatin $(5 \mu \mathrm{M})$ in the presence or absence of 3-MA $(10 \mathrm{mM})$. After $24 \mathrm{~h}$ of treatment, the cells were harvested using trypsin and were then washed twice in PBS. Subsequently, they were treated with Annexin V/fluorescein isothiocyanate (FITC) $(0.5 \mu \mathrm{g} / \mathrm{ml}$ final concentration) combination, PI ( $2 \mu \mathrm{g} / \mathrm{ml}$ final concentration), and apoptosis detection kit (Assay Designs), for $10 \mathrm{~min}$ at room temperature. The cells were then immediately examined using a flow cytometer (FACSVantage flow cytometer; Becton-Dickinson Immunocytometry System, San Jose, CA, USA) following the addition of $250 \mu \mathrm{l}$ binding buffer. Analysis was performed using CellQuest software (Becton-Dickinson, Franklin Lakes, NJ, USA). To quantitatively analyze autophagy, the cells were plated in 6-well plates and incubated for $24 \mathrm{~h}$. The cells were then treated with cisplatin $(5 \mu \mathrm{M})$ in the presence or absence of 3-MA $(10 \mathrm{mM})$. After $24 \mathrm{~h}$ of treatment, the cells were stained with $\mathrm{AO}(1 \mu \mathrm{g} / \mathrm{ml})$ for $15 \mathrm{~min}$ at $37^{\circ} \mathrm{C}$, harvested with trypsin and subsequently washed with PBS. We then added $500 \mu \mathrm{l}$ FACS buffer (1\% FBS in PBS). The cells were immediately counted by flow cytometry (FACSVantage flow cytometer) after addition of $250 \mu \mathrm{l}$ binding buffer. Analysis was performed using CellQuest software.

Statistical analysis. The experiment was performed thrice independently, with the means \pm standard deviation (SD) recorded. Results were analyzed using the Student's t-test. $\mathrm{p}<0.05$ was considered to indicate a statistically significant difference.

\section{Results}

Detection of wild-type p53 expression in the NCI-H460 and NCI-H1299 cell lines. We confirmed wild-type p53 protein expression after treatment of NCI-H460 cell lines with $5 \mu \mathrm{M}$ cisplatin in the presence or absence of 3-MA (10 mM), an autophagy-specific inhibitor. We found that wild-type p53 was not expressed after treatment with $5 \mu \mathrm{M}$ cisplatin in the presence or absence of 3-MA in NCI-H1299 cell lines (Fig. 1).

Cell viability decreases by low-dose cisplatin in $H 460$ and H1299 cell lines. We investigated cell death by treating both cell lines with $5 \mu \mathrm{M}$ cisplatin in the presence or absence of $10 \mathrm{mM}$ 3-MA. The cell viability was measured by an MTT 

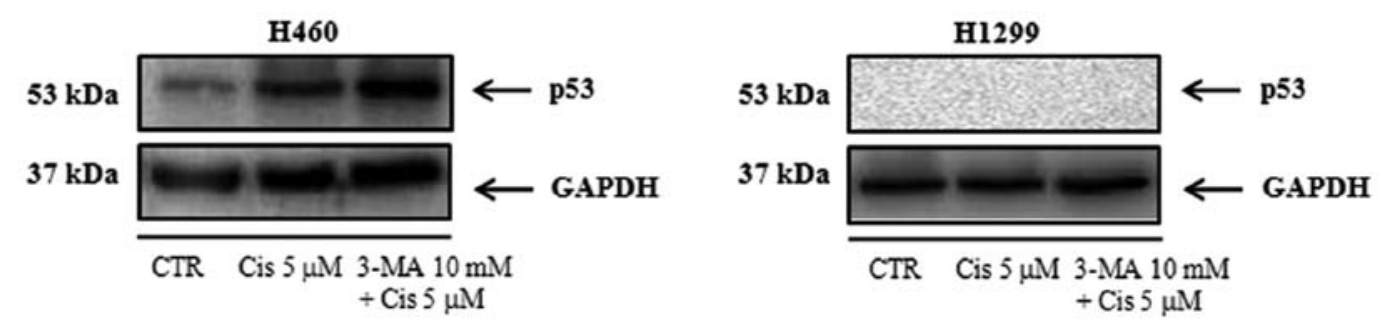

Figure 1. Expression of p53 protein in H460 and H1299 cells. H460 and H1299 cells were treated with $5 \mu$ M cisplatin with or without 10 mM 3-MA for 24 h. Expression of 53 protein was confirmed by western blot analysis. GAPDH was used as an equal loading control.
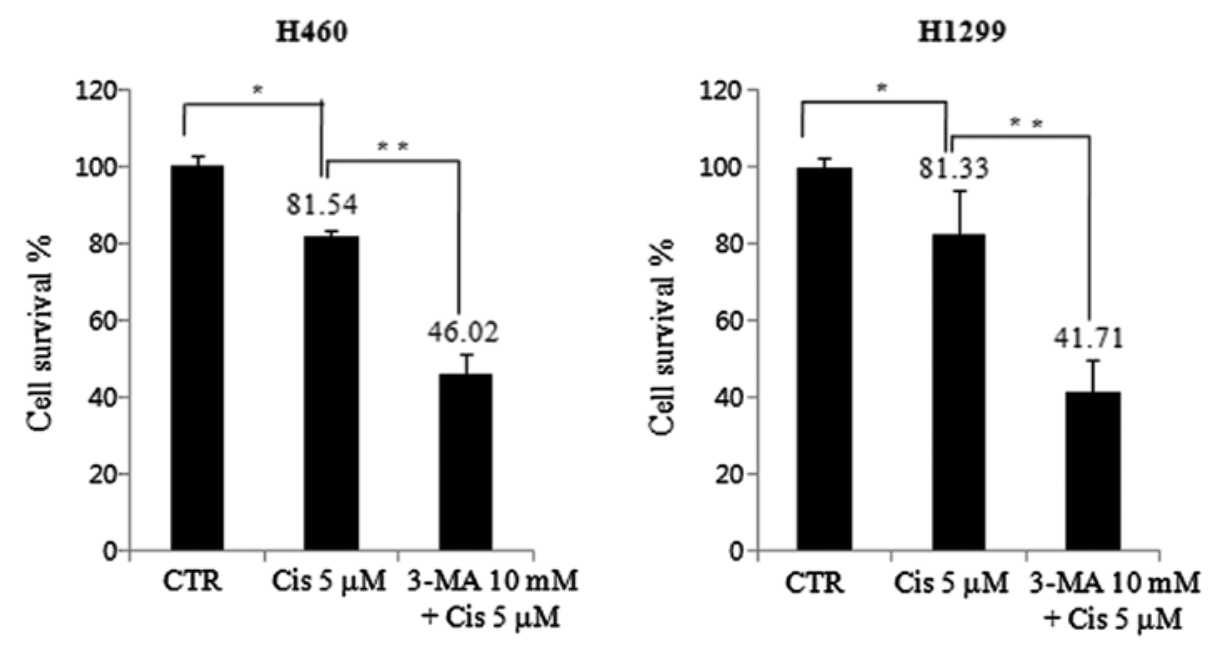

Figure 2. Effect of cisplatin-induced cell death on $\mathrm{H} 460$ and H1299 cells. $\mathrm{H} 460$ and H1299 cells were seeded at 5x10 4 cells/well in 48 -well flat-bottomed plates and incubated overnight at $37^{\circ} \mathrm{C}$. They were then treated with $5 \mu \mathrm{M}$ cisplatin for $24 \mathrm{~h}$. The viability of the untreated cells was regarded as $100 \%$. Cell viability was measured by an MTT assay. Values represent means $\pm \operatorname{SD}(\mathrm{n}=3) .{ }^{*} \mathrm{p}<0.05,{ }^{* *} \mathrm{p}<0.01$.

assay. For the $\mathrm{H} 460$ cell line, cell viability in the group treated with $5 \mu \mathrm{M}$ cisplatin was $81.54 \%$, lower than that in the control group. This further decreased to $46.02 \%$ in the presence of $10 \mathrm{mM}$ 3-MA. Cell viability was further decreased by autophagy inhibition. In the H1299 cell line, cell viability in the group treated with $5 \mu \mathrm{M}$ cisplatin was $81.33 \%$, lower than that in the control group, and it further decreased to $41.71 \%$ in the presence of $10 \mathrm{mM} 3-\mathrm{MA}$. However, there was no difference in cell viability between cisplatin and 3-MA pretreatment in H460 and H1299 cell lines (Fig. 2).

Observation of morphological characteristics and biodynamics analysis of autophagy. After $24 \mathrm{~h}$, autophagosomes, the morphological characteristics of autophagy, were observed in cells treated with $5 \mu \mathrm{M}$ cisplatin. After $48 \mathrm{~h}$, the number of autophagosomes observed increased. Cells treated with $20 \mu \mathrm{M}$ cisplatin showed low autophagosome formation after $24 \mathrm{~h}$ compared with those treated with $5 \mu \mathrm{M}$ cisplatin. After $48 \mathrm{~h}$, no autophagosomes were observed in the cells treated with $20 \mu \mathrm{M}$ cisplatin.

LC3-I is present in the cytoplasm, and LC3-II, which is formed during autophagy, is present in the autophagosome. LC3-I is converted to LC3-II during the initiation of autophagy. Thus, LC3 is a marker of autophagosome formation. In the $\mathrm{H} 460$ cell lines treated with $5 \mu \mathrm{M}$ cisplatin, autophagosomeincorporated LC3-II protein expression increased from 12 to 24 and $48 \mathrm{~h}$, compared with the control. Therefore, autophagy increased during this period. However, in the H1299 cell lines, LC3-II protein expression did not change compared with the control. Therefore, in the H1299 cell lines, no change in autophagy was observed (Fig. 3).

Quantitative measurements of apoptosis and autophagy in cisplatin-treated NCI-H460 and NCI-H1299 cell lines. For quantitative measurement of apoptosis, we stained the cell lines with Annexin V and PI after treatment with $5 \mu \mathrm{M}$ cisplatin for $24 \mathrm{~h}$, followed by analysis using flow cytometry. A 5.9-fold increase in apoptosis was observed in the $\mathrm{H} 460$ cell lines of wild-type p53. In the control group, $2 \%$ more apoptosis was observed and $11.18 \%$ more apoptosis was observed in the group treated with $5 \mu \mathrm{M}$ cisplatin. In the $\mathrm{H} 1299$ cell lines with null-type p53, apoptosis increased by 7.62 -fold. In the control group, a 3.86\% increase in apoptosis was observed along with a $29.45 \%$ increase in the $5 \mu \mathrm{M}$ cisplatin group. Thus, more apoptosis was observed in the p53 null cell line groups than in the p53 wild-type cell line groups (Fig. 4).

For quantitative measurement of autophagy, we analyzed AVOs by flow cytometry in the cell lines treated with $5 \mu \mathrm{M}$ cisplatin for $24 \mathrm{~h}$. A 12.49 -fold increase in autophagy was observed in the H460 cell lines of wild-type p53. In the control group, autophagy increased by $3.3 \%$, and in the $5 \mu \mathrm{M}$ cisplatin group, it increased by $41.22 \%$. A 6.67 -fold increase was observed in the H1299 cell lines of null-type p53. In the control group, autophagy increased by $7.4 \%$, and in the $5 \mu \mathrm{M}$ 
A
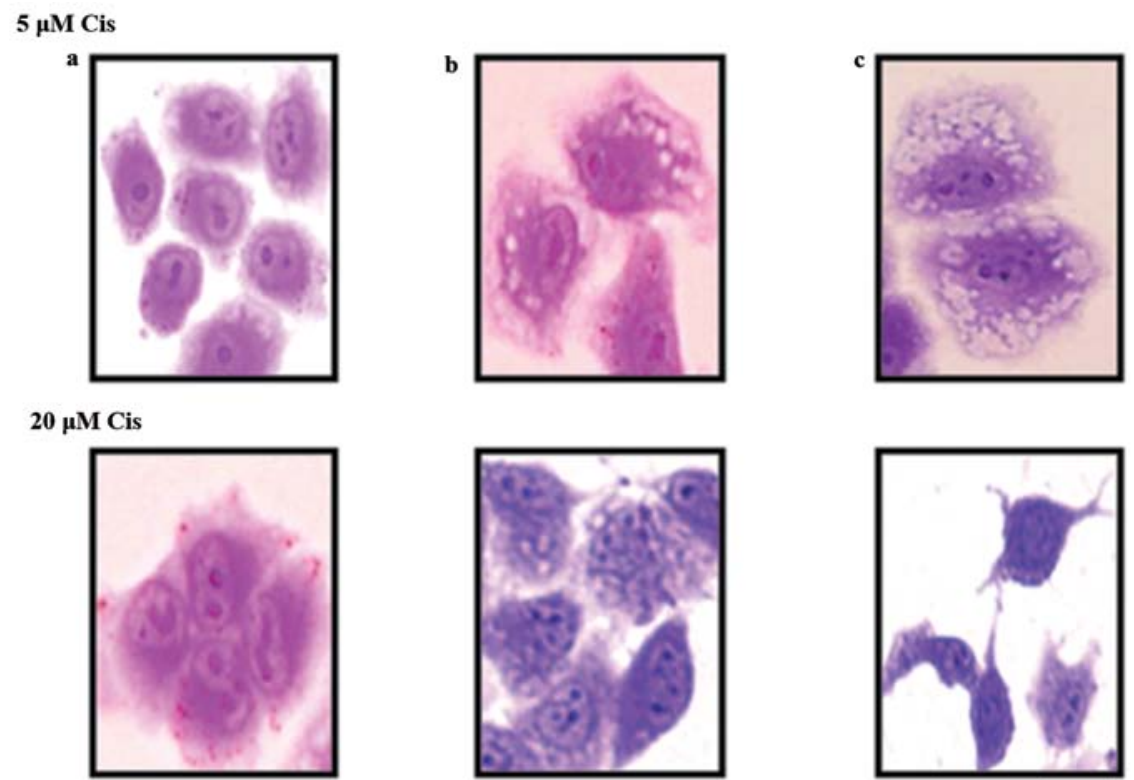

Control

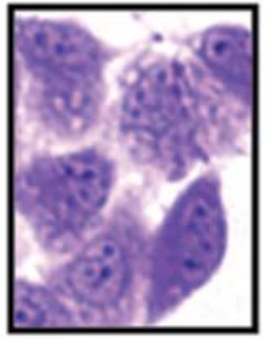

24 h

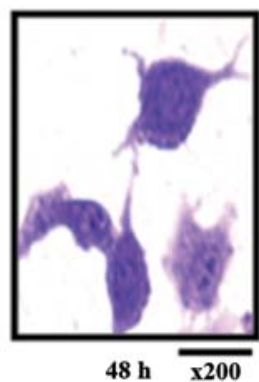

B
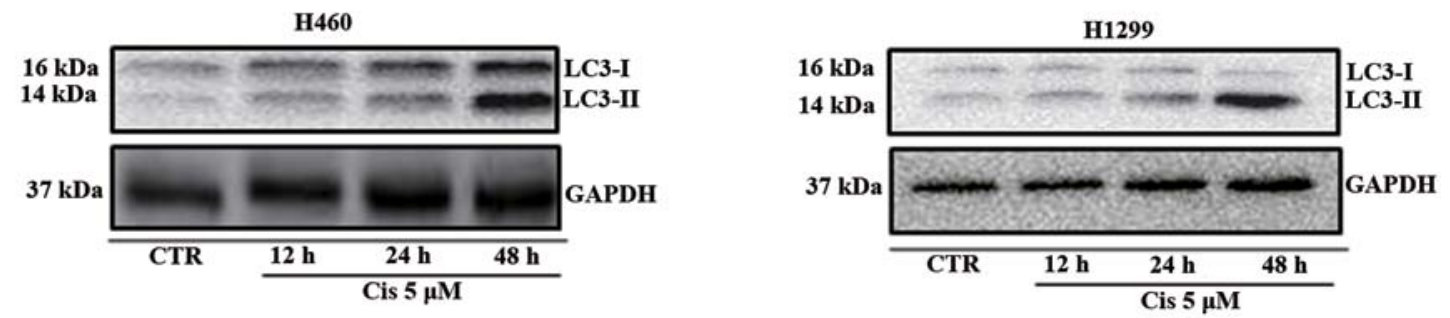

Figure 3. Morphological phenomena and LC3-II protein expression of autophagy in cisplatin-treated lung cancer cells. (A) Direct observation of cells treated with 5 and $20 \mu \mathrm{M}$ cisplatin for 24 and $48 \mathrm{~h}$ in H460 cells. Magnification, x200. Samples were examined under a light microscope. (B) H460 and H1299 cells were treated with $5 \mu \mathrm{M}$ cisplatin for 12,24 and $48 \mathrm{~h}$. Expression of LC3-II protein was detected by western blot analysis. GAPDH was used as an equal loading control.

A

H460

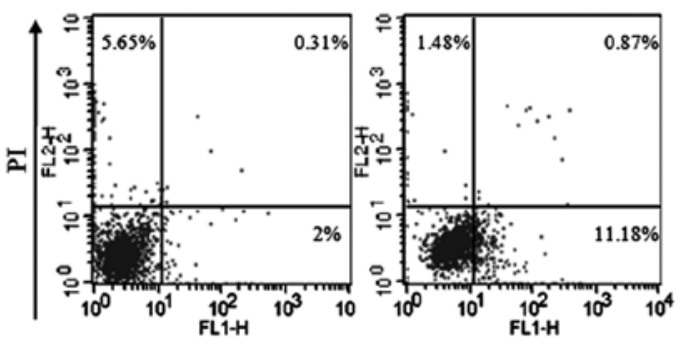

H1299

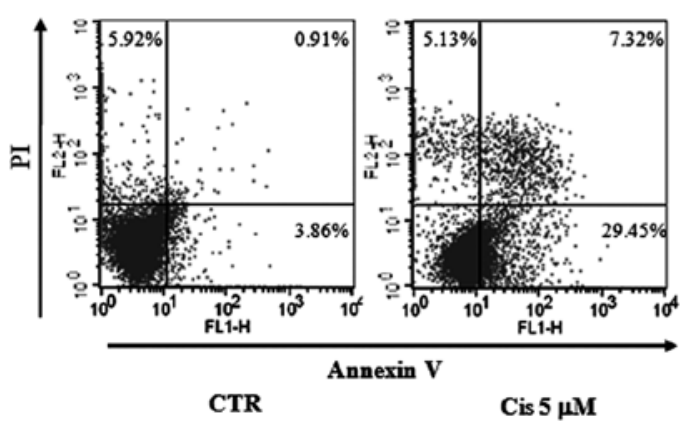

B
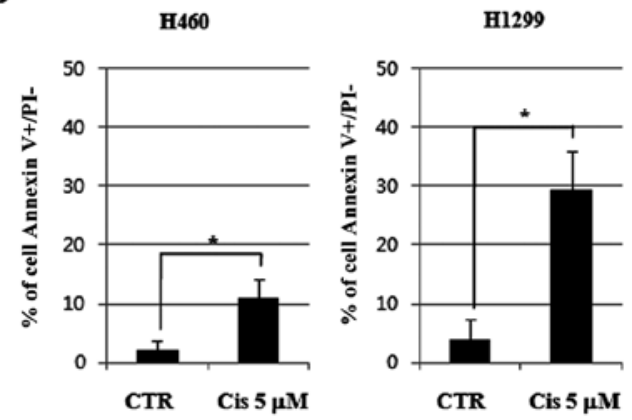

Figure 4. Increase in apoptosis with low-dose cisplatin treatment in H460 and H1299 cells. (A) H460 and H1299 cells were treated with $5 \mu$ M cisplatin for $24 \mathrm{~h}$. Annexin V and PI staining were used to identify apoptotic and necrotic cells. FACS analysis of apoptotic cells using Annexin V and PI indicated that the Annexin V (+) and PI (-) stained populations were apoptotic cells. Representative FACS plots are shown; stains were performed in 3 independent experiments with similar results unless otherwise indicated. (B) Apoptotic cell population is expressed as a \% of the total cell population. * $\mathrm{p}<0.05$. 
$\mathbf{A}$
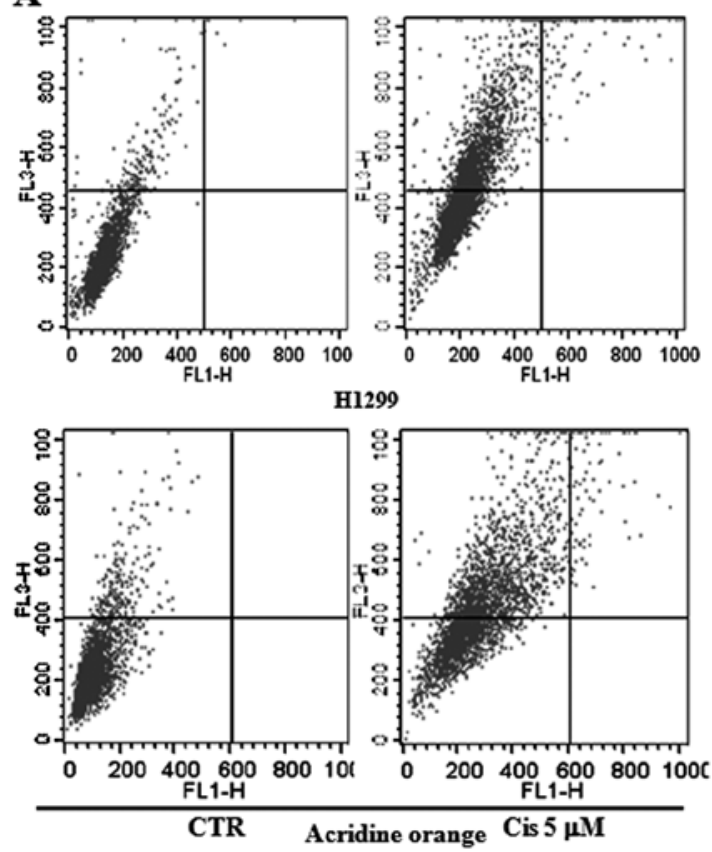

B
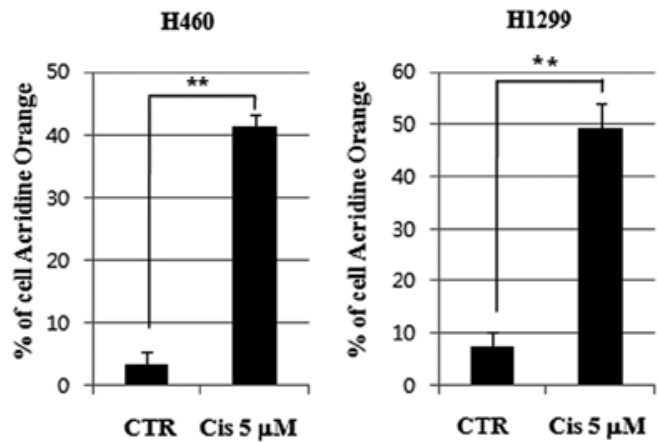

Figure 5. Increase in apoptosis in low-dose cisplatin-treated H460 and H1299 cells. (A) H460 and H1299 cells were treated with $5 \mu$ M cisplatin for 24 h. Acridine orange staining was used to identify autophagic cells by FACS. Representative FACS plots are shown. Staining was performed in 3 independent experiments with similar results, unless otherwise indicated. (B) Acridine orange-positive cell population is expressed as a $\%$ of the total cell population. ${ }^{* *}$ p $<0.01$.

$\mathbf{A}$

H460
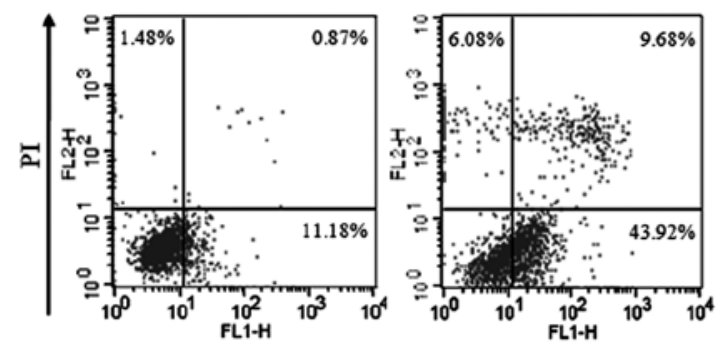

H1299
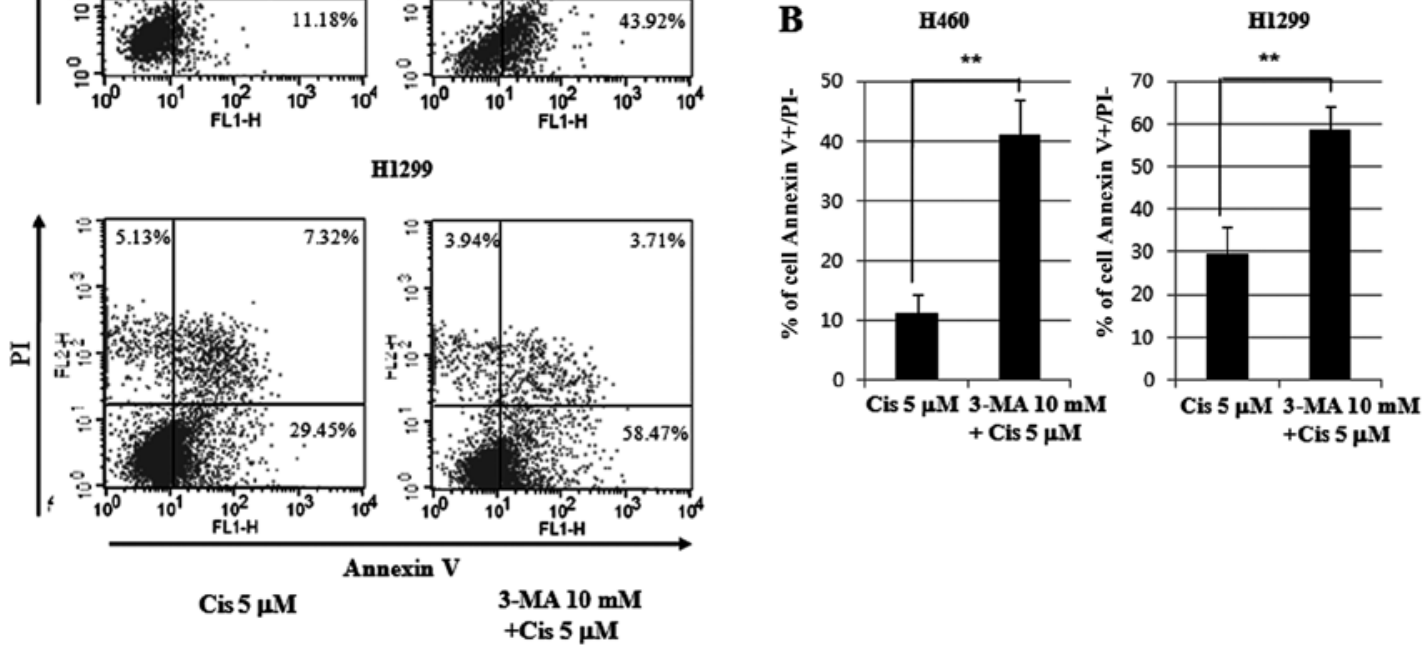

Figure 6. Increase in apoptosis in H460 and H1299 cell lines treated with low-dose cisplatin and pretreated with 3-MA. (A) H460 and H1299 cells were treated with $5 \mu \mathrm{M}$ cisplatin for $24 \mathrm{~h}$. Annexin V and PI staining were used to identify apoptotic and necrotic cells. FACS analysis of apoptotic cells using Annexin-V and PI indicated that the Annexin-V (+) and PI (-) stained populations were apoptotic cells. Representative FACS plots are shown. Staining was performed in 3 independent experiments with similar results, unless otherwise indicated. (B) Apoptotic cell population is expressed as a $\%$ of the total cell population. ${ }^{* *}$ p $<0.01$.

cisplatin group, it increased by $49.39 \%$. Therefore, autophagy was induced more in the p53 wild-type cell lines than in the p53 null-type cell lines (Fig. 5). These results suggest that cisplatin-treated p53 wild-type cells play a role in inducing autophagy and inhibiting apoptosis.
Quantitative measurements of cisplatin-induced apoptosis and autophagy after 3-MA treatment in H460 and H1299 cell lines. The $5 \mu \mathrm{M}$ cisplatin-treated cell lines with 3-MA pretreatment for $24 \mathrm{~h}$ were stained with Annexin V and PI, and subjected to flow cytometry. A 3.72-fold increase in apop- 
A

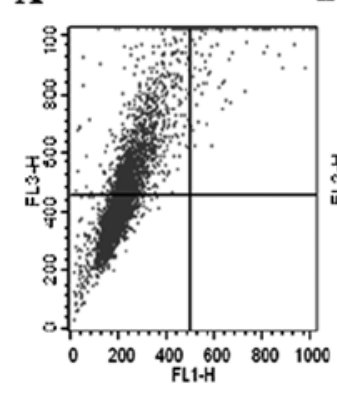

H460

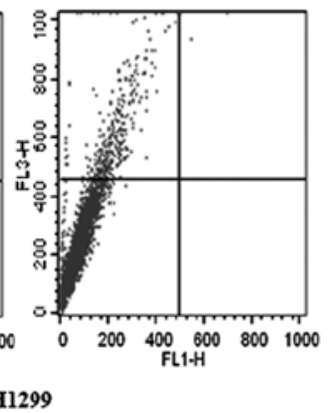

.
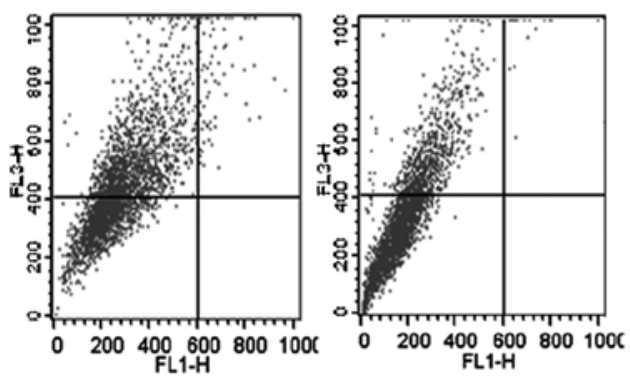

Acridine orange

Cis $5 \mu \mathrm{M}$
3-MA $10 \mathrm{mM}$
$+\mathrm{C}$ is $5 \mu \mathrm{M}$
B
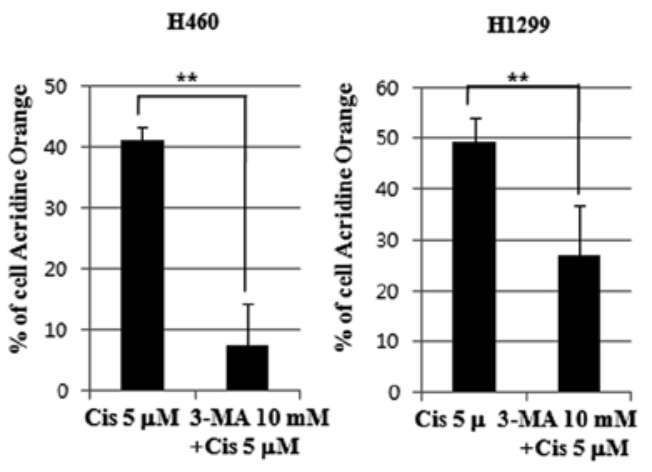

Figure 7. Reduction in autophagy in H460 and H1299 cell lines treated with low-dose cisplatin and pretreated with 3-MA. (A) H460 and H1299 cells were treated with $5 \mu \mathrm{M}$ cisplatin for $24 \mathrm{~h}$. Acridine orange staining was used to identify autophagic cells by FACS. Representative FACS plots are shown. Staining was performed in 3 independent experiments with similar results, unless otherwise indicated. (B) Acridine orange-positive cell population is expressed as a $\%$ of the total cell population.

tosis in the p53 wild-type H460 cell line was observed. An $11.18 \%$ increase was observed in the $5 \mu \mathrm{M}$ cisplatin group, and a $43.92 \%$ increase was observed in the $5 \mu \mathrm{M}$ cisplatin group that was pretreated with 3-MA. In the H1299 cell line of p53 null-type, a 1.98-fold increase in apoptosis was observed. A $29.45 \%$ increase was observed in the $5 \mu \mathrm{M}$ cisplatin group, and a $58.47 \%$ increase was observed in the $5 \mu \mathrm{M}$ cisplatin group that was pretreated with 3-MA. The p53 wild-type cell lines induced twice as much apoptosis as the p53 null-type cell lines (Fig. 6).

To measure autophagy, the $5 \mu \mathrm{M}$ cisplatin-treated cell lines with 3-MA pretreatment for $24 \mathrm{~h}$ were again stained with Annexin V and PI, and subjected to flow cytometry. A 5.57-fold decrease in autophagy in the p53 wild-type H460 cell line was observed. A $41.22 \%$ decrease was observed in the $5 \mu \mathrm{M}$ cisplatin group, and a $7.4 \%$ decrease was observed in the $5 \mu \mathrm{M}$ cisplatin group that was pretreated with 3-MA. In the H1299 cell line of p53 null-type, a 1.83-fold decrease in autophagy was observed. A $49.39 \%$ decrease was observed in the $5 \mu \mathrm{M}$ cisplatin group, and a $26.88 \%$ decrease was observed in the $5 \mu \mathrm{M}$ cisplatin group that was pretreated with 3-MA. The p53 wild-type cell lines reduced autophagy 3-fold more than the p53 null-type cell lines (Fig. 7). Therefore, we concluded that p53 inhibited low-dose cisplatin-induced autophagy and induced apoptosis.

\section{Discussion}

At the time of diagnosis, many cases of lung cancer are considered inoperable due to infiltration of the surrounding tissue by cancer cells. Treatment of lung cancer by chemotherapy and radiation therapy presents difficulties, and, therefore, prognosis for lung cancer is often bleak. However, the discovery and subsequent adoption of cisplatin in the 1980s helped improve survival rates of patients with lung cancer. New second-generation anticancer drugs were developed in the 1990 s, but they have yet to produce satisfactory results.

Research has recently started to focus on targeted therapies. However, anticancer treatment is still restricted to cisplatin for local progression of lung cancer, despite the fact that the use of cisplatin causes suffering and often results in treatment being stopped due to toxicity, low white blood cell counts, thrombocytopenia symptoms, vomiting and neurological toxicity, among other side-effects.

Therefore, some patients choose to undergo anticancer treatment using non-cisplatin based chemotherapy for local lung cancer progression. However, these are often less effective than cisplatin treatments. Furthermore, targeted therapies often only show significant results in some patients. Many clinicians regulate the dose intensity of cisplatin so as to reduce the effects of toxicity. However, low-dose treatments sometimes have minor effects.

Research has shown that autophagy affects signal transduction. Autophagy and apoptosis have already prompted much research in cancer. However, the role of autophagy in cell survival or apoptosis remains to be clarified. Autophagy assists cell survival by helping cancer cells resist radiation therapy, chemotherapy and low-nutrient environments. However, apoptosis helps contribute to cancer cell suicide and death on exposure to chemotherapy or radiation. These paradoxical 
features are a point of debate as to whether autophagy is a friend or foe (15-17). Without autophagy, genome damage caused by metabolic stress is mostly unhindered. Furthermore, autophagic defects are associated with increased tumorigenesis. However, it is not possible to conclude that cancer cell extirpation occurs via autophagy induction or inhibition (18). The number of discrepancies related to the role of autophagy in cell death and cancer presents difficulties. Animal experiments have shown that autophagy increases tumor cell survival during periods of metabolic stress. However, it has also been shown to prevent tumors, necrosis and inflammation. In this case, the tumor-suppressing effects of autophagy had a greater effect than the cell survival mechanisms promoted by autophagy. In other animal experiments, treatment-induced apoptosis and tumor extinction increased when chloroquine was used to inhibit autophagy. The combination therapy of chloroquine and the histone deacetylase inhibitor SAHA has a multiplier effect in killing imatinib-refractory chronic myeloid leukemia cells. This protective function supports the therapeutic use of autophagic inhibitors for cancer treatment (19-22). Autophagosomes in the cytoplasm were observed at 24 and $48 \mathrm{~h}$ in cell lines treated with low-dose $(5 \mu \mathrm{M})$ cisplatin in a previous study. The conversion of LC3-I into LC3-II within the cytoplasm increases over time. In this study, we investigated the role of p53 in autophagy induction in wild-type p53 NCI-H460 cell lines and null-type p53NCI-H1299 cell lines treated with low-dose cisplatin. No difference was observed in the viability of H460 and H1299 cells treated with cisplatin and pretreated with 3-MA. However, a difference between apoptosis and autophagy was observed. After treatment with $5 \mu \mathrm{M}$ cisplatin, apoptosis increased by 5.59-fold in the wild-type p53 H460 cell line compared to the control group, and apoptosis in the null-type p53 H1299 cell lines increased by 7.62-fold.

For autophagy, a 12.49 -fold increase after $5 \mu \mathrm{M}$ cisplatin treatment was observed in the wild-type p53H460 cell line compared to the control group, and autophagy in the null-type p53 H1299 cell line increased by 6.69 -fold. Autophagic activity in the null-type p53 H1299 cell lines was twice that of the wild-type p53 H460 cell lines. On the basis of this result, we conclude that wild-type p53 has a role in autophagic induction and apoptosis inhibition when treated with low-dose cisplatin. In addition, after 3-MA pretreatment and $5 \mu \mathrm{M}$ cisplatin treatment, apoptosis increased by 3.92-fold in the wild-type p53 H460 cell line compared to the control group. In the null-type p53 H1299 cell line, apoptosis increased by 1.98 -fold. Apoptotic activity in the null-type p53 H1299 cell lines was twice that of the wild-type p53 H460 cell lines. After 3-MA pretreatment and $5 \mu \mathrm{M}$ cisplatin treatment, autophagy decreased 5.57-fold in the wild-type p53H460 cell lines and decreased by 1.83 -fold in the null-type p53 H1299 cell lines. Autophagic activity decreased thrice as much in the null-type than in the wild-type p53 cell line. This result suggests that p53-induced apoptosis also inhibited autophagy induced by low-dose cisplatin. However, this result does not clarify the role of p53. Therefore, further studies are required to ascertain how 3-MA-pretreated and cisplatin-treated cells affect apoptosis and autophagy.

\section{Acknowledgements}

This study was supported by a grant from the Korea Health 21 R\&D Project (Ministry of Health, Welfare and Family Affairs, Republic of Korea, A010251).

\section{References}

1. Ferreira CG, Span SW, Peters GJ, et al: Chemotherapy triggers apoptosis in a caspase-8-dependent and mitochondria-controlled manner in the non-small cell lung cancer cell line NCI-H460. Cancer Res 60: 7133-7141, 2000.

2. Fukuoka M, Yano S, Giaccone G, et al: Multi-institutional randomized phase II trial of gefitinib for previously treated patients with advanced non-small-cell lung cancer. J Clin Oncol 21: 2237-2246, 2003.

3. Kundu M and Thompson CB: Autophagy: basic principles and relevance to disease. Annu Rev Pathol 3: 427-455, 2008.

4. Levine B and Yuan J: Autophagy in cell death: an innocent convict? J Clin Invest 115: 2679-2688, 2005.

5. Hara T, Nakamura K, Matsui M, et al: Suppression of basal autophagy in neural cells causes neurodegenerative disease in mice. Nature 441: 885-889, 2006.

6. Nakai A, Yamaguchi O, Takeda T, et al: The role of autophagy in cardiomyocytes in the basal state and in response to hemodynamic stress. Nat Med 13: 619-624, 2007.

7. Roy S and Debnath J: Autophagy and tumorigenesis. Semin Immunopathol 32: 383-396, 2010.

8. Kondo Y, Kanzawa T, Sawaya R and Kondo S: The role of autophagy in cancer development and response to therapy. Nat Rev Cancer 5: 726-734, 2005.

9. Rosenfeldt MT and Ryan KM: The role of autophagy in tumour development and cancer therapy. Expert Rev Mol Med 11: e36, 2009.

10. Giaccia AJ and Kastan MB: The complexity of p53 modulation: emerging patterns from divergent signals. Genes Dev 12: 2973-2983, 1998.

11. Levine B and Abrams J: p53: the Janus of autophagy? Nat Cell Biol 10: 637-639, 2008.

12. Riley T, Sontag E, Chen P and Levine A: Transcriptional control of human p53-regulated genes. Nat Rev Mol Cell Biol 9: 402-412, 2008.

13. Zilfou JT and Lowe SW: Tumor suppressive functions of p53. Cold Spring Harb Perspect Biol 1: a001883, 2009.

14. Green DR and Kroemer G: Cytoplasmic functions of the tumour suppressor p53. Nature 458: 1127-1130, 2009.

15. Karantza-Wadsworth V and White E: Role of autophagy in breast cancer. Autophagy 3: 610-613, 2007.

16. Levine B: Unraveling the role of autophagy in cancer. Autophagy 2: 65-66, 2006

17. Morselli E, Galluzzi L, Kepp O, et al: Anti- and pro-tumor functions of autophagy. Biochim Biophys Acta 1793: 1524-1532, 2009.

18. Maiuri MC, Tasdemir E, Criollo A, et al: Control of autophagy by oncogenes and tumor suppressor genes. Cell Death Differ 16: 87-93, 2009.

19. Vogelstein B, Lane D and Levine AJ: Surfing the p53 network. Nature 408: 307-310, 2000.

20. Vousden KH and Lane DP: p53 in health and disease. Nat Rev Mol Cell Biol 8: 275-283, 2007.

21. Feng Z, Zhang H, Levine AJ and Jin S: The coordinate regulation of the p53 and mTOR pathways in cells. Proc Natl Acad Sci USA 102: 8204-8209, 2005.

22. Tasdemir E, Maiuri MC and Galluzzi L: Regulation of autophagy by cytoplasmic p53. Nat Cell Biol 10: 676-687, 2008.

23. Maiuri MC, Galluzzi L, Morselli E, et al: Autophagy regulation by p53. Curr Opin Cell Biol 22: 181-185, 2010. 